\title{
The Mammalian Genome special issue on ageing
}

\author{
Paul K. Potter ${ }^{1}$
}

Published online: 10 June 2016

(c) Springer Science+Business Media New York 2016

With the ever-increasing numbers of elderly people across the globe, there is a growing recognition of the benefits of the study of the process of ageing and the genetic and environmental determinants of late-onset disease. Developing disease model systems that allow us to explore and test mechanisms of late-onset disease, including the impact of the ageing process, will be critical. While a number of models of age-related disease are early onset or induced and develop a rapid disease, the need for disease models that emulate the chronic and/or late-onset aspects of human patients is increasingly recognised. Not only will such models provide us with mechanistic insights into the pathophysiology of late-onset disease, but they will be important vehicles for the development and assessment of novel therapeutics.

The study of ageing itself is critical to our understanding of its impact on health. Lewis et al. outline how the genetic interrogation of the long-lived naked mole rat may help us understand its resistance to many aspects of ageing, and Kaeberlein et al. review work on the opportunities for geroscience in studying domestic dogs. Ageing can be modulated and in particular this has an effect on healthspan, not simply longevity. Andrez Bartke outlines the influence of growth hormone signalling and Van Cauwenberghe et al. describe how mimetics of caloric restriction, an intervention thought to be beneficial to ageing, may also have potential in the treatment of neurodegenerative disease. Parry and Narita relate the breadth

Paul K. Potter

p.potter@har.mrc.ac.uk

1 The MRC Harwell Institute, Harwell Science and Innovation Campus, Harwell OX11 ORD, UK of work on the regulation of cellular senescence which is thought to play a central role in ageing.

Ageing has a direct effect on tissues and physiological systems that in turn influence the development of disease. Banks et al. describe the changes to circadian rhythm with age and their consequences. In a similar vein, the theme of circadian rhythm alterations with ageing is picked up by Goljanek-Whysall et al. in their review of skeletal muscle dysfunction. Chris Jones highlights the need for a better understanding of the changes in platelet function with age, whilst Adams et al. review the genetics of ageing bone. Diot et al. outline the relationships between mitochondria and ageing, with Krauss and de Haan describing the influence ageing has on stem cells and the subsequent decline in homeostasis. The consequences of the effects of ageing on the blood-brain barrier is covered by Gorle et al., whilst Sacitharan and Vincent look at the effect of ageing on the processes influencing osteoarthritis. Aiken et al. discuss the long-term and transgenerational consequences of early-life environment. This not only has implications for human health but also for model studies, as metadata such as age and metabolic health of dams may need to be considered in long-term ageing experiments.

Finally, there are two articles that highlight the need for research infrastructures to support the study of ageing in mice (Wells and Bellantuono) and discuss the progress that is now being made towards such facilities in Europe (Raess et al.). The need for centres of expertise in the care and welfare of ageing mice, as well as the ability to deliver comprehensive phenotypic analysis of models of disease and animals undergoing treatments, is not to be underestimated. The all-pervasive effect of ageing on the majority of physiological systems underlines the need for a broad systems analysis of disease models and the outcomes of interventions. 
We can look forward with excitement to the next few years in which progress with developments of novel disease models alongside a greater understanding of ageing and its effects on health will bring both continuing mechanistic insights into late-onset disease along with tangible benefits in translational studies and therapeutic opportunities. This Special Issue highlights the path we are taking and the goals that the community aspires to. It only remains for me to thank all those involved in the preparation of this special issue: the guest editors Ilaria Bellantuono, Colin Selman, Ron Korstanje, Claude Libert, Gerald de Haan, and especially Louise Tinsley for her forbearance and guidance. 